\title{
Trastuzumab as Intellectual, Medical and Economical Challenge
}

\author{
Christoph C. Zielinski \\ Clinical Division of Oncology, Department of Medicine I, Medical University Vienna, Austria
}

The biologic concept of breast cancer has shifted dramatically from the assumption of a largely local to a potentially systemic disease during the past 30 years. It was quite logical to have therapeutic consequences follow this shift in paradigm. From the very outset of this revolutionary journey, two principles of treatment were followed: the quite obvious, but only developing cytotoxic therapy and endocrine treatment, which was based upon the very visionary concept of hormone dependence of cancer followed since the end of the 19th century. The latter reached an impressive peak by the award of the Nobel Prize to Dr. Huggins in 1966. From today's point of view, it was only logical to test for the correctness of the concept of adjuvant therapy and thus reduce the extension of surgical intervention and the incidence of disease recurrence, thereby prolonging survival. As we all know, adjuvant treatment and reduction of surgical treatment in patients with breast cancer was mainly developed by the superb work and genius of Drs. Bernard Fisher, Gianni Bonadonna and Umberto Veronesi. It proved to be tremendously successful leading to an impressive reduction in disease recurrence and a significant increase in survival [1], thus impressively proving the correctness of the pioneers' assumption. The introduction of anthracyclines and taxanes on one hand and of aromatase inhibitors and LH-RH agonists on the other hand, led to a further amelioration of results, as compared to the initial use of the cytotoxic combination of cyclophosphamide, methotrexate and fluorouracil as well as endocrine intervention by tamoxifen which already had proven to be highly efficacious in individual trials as well as large metaanalyses. However, progress occurred only slowly over the years which was due to the biology of the disease, the necessity to recruit large patient populations in order to arrive at meaningful results and, the truth be told, also our lack of understanding of regulatory mechanisms influencing breast cancer development, cell proliferation and metastatic spread thus limiting our possibilities of intervention to two options, endocrine or cytotoxic, no matter how intricate the underlying concepts might have been.

This era came to an end heralded by the discovery of a detrimental prognosis in a subpopulation of patients who overexpressed the Her-2/neu protein [2] which was the product of the erb-B2 gene from the family of epidermal growth factor receptors (EGFR). The generation and development of an anti-Her-2/neu monoclonal antibody later called trastuzumab led to impressive results in patients with metastatic breast cancer overexpressing the Her-2/neu protein and prolonged significantly disease-free as well as overall survival in combination with cytotoxic chemotherapy, as compared to treatment with chemotherapy alone [3]. In fact, the advent of trastuzumab has reversed the detrimental prognostic factor of Her-2/ neu overexpression and generated a treatment option leading to an impressive and previously unseen duration of survival in advanced disease.

With this observation, the stage was set for clinical trials testing the efficacy of trastuzumab administered over a period of one year in the adjuvant setting. Primarily three randomized trials were available, out of which two were identical in design [4] and the third different in the choice of cytotoxic treatment, the sequence of trastuzumab administration relative to the administration of cytotoxic treatment as well as the inclusion of patients with positive and negative lymph node [5]. Although trials differed in the mentioned variables, results were surprisingly equivocal and overwhelming in the reported results in that the addition of trastuzumab to cytotoxic treatment lead to an enormous reduction in recurrence of disease by more than $50 \%$ in patients with Her-2/neu overexpressing breast tumors $[4,5]$ irrespective of lymph node involvement [5]. Although the period of observation was short, overall survival was also significantly reduced. The scientific community was overwhelmed by the dimension of the results unprecedented in their impact upon the course of breast cancer in the said pop-

\begin{tabular}{|c|c|}
\hline KARGER & (C) 2006 S. Karger GmbH, Freiburg \\
\hline $\begin{array}{l}\text { Fax +49 } 7614520714 \\
\text { E-mail Information@Karger.de } \\
\text { www.karger.com }\end{array}$ & $\begin{array}{l}\text { Accessible online at: } \\
\text { www.karger.com/brc }\end{array}$ \\
\hline
\end{tabular}

Prof. Dr. Christoph Zielinski

Universitätsklinik für Innere Medizin I, Klinische Abteilung für Onkologie

Währinger Gürtel 18-20, 1090 Wien, Austria

Tel. +43 1 40400-4445, Fax -4428

E-mail christoph.zielinski@meduniwien.ac.at

www.meduniwien.ac.at/krebszentrum 
ulation of patients. After the Annual Meeting of the American Society of Clinical Oncology where these studies were presented and their expedited publication in the New England Journal of Medicine, the results of the mentioned studies soon proved to constitute a major milestone not only for medical care of patients with Her-2/neu overexpressing breast cancer, but also for the readiness of health systems to provide patients with optimal standards of care. With annual costs of trastuzumab being about $€ 30-35,000$,- per person suffering from a devastating, yet frequently occurring disease, health systems soon found themselves in a dilemma: Out of 50,000 German women who suffer from early breast cancer each year, around 10,000 have an overexpression of HER-2/neu on tumor cells thus qualifying for combined treatment with cytotoxic chemotherapy and trastuzumab [6]. After a short period of uninformed or helpless silence, health authorities often played the problem down in public by pointing out that only a minor population of patients would benefit from trastuzumab treatment, that the efficacy of trastuzumab in the adjuvant treatment of breast cancer would the exaggerated, or that trastuzumab would have serious, as yet unknown side effects [7]. Another attempt to withhold trastuzumab from patients included the protracted examination and analysis of known and published data by regulatory bodies in an attempt to gain time and save money at least for some period of time [8]. However, adopted strategies differed from country to country: Whereas, e.g. Viennese health authorities took over the costs of treatment of trastuzumab in the adjuvant setting after a very short discussion with qualified experts, many patients from Germany had to appeal to courts to decide about their right for access to trastuzumab in the adjuvant setting. There is little doubt that the reluctance of authorities in countries with socialized health care systems to provide qualified patients with access to trastuzumab in the adjuvant setting will falter under the pressure from scientific evidence and patients' rights groups.

However, new strategies arise which suggest that a much shorter use of trastuzumab than the previously reported period of one year might be similarly effective: a very recent study from Finland presented in abstract form showed that the administration of only 9 weekly cycles of trastuzumab resulted in a reduction in hazard ratio of recurrence of 0.46 which was comparable to the reduction of recurrence as the previously reported extended use of trastuzumab for one year, although this was not tested in a head-to-head comparison [9]. The proof of concept of the efficacy of a limited use of trastuzum$\mathrm{ab}$ in the adjuvant setting of early breast cancer would not only teach us a new lecture in the understanding of the biology of breast cancer and the history of treatment of this disease, but also alleviate health systems to a certain degree from the exploding costs connected with the use of the substance even in a subfraction of women with early breast cancer. Only controlled clinical trials which are strongly encouraged in this context can pave the way to this goal.

\section{References}

1 EBCTCG: Effects of chemotherapy and hormonal therapy for early breast cancer on recurrence and 15-year survival: an overview of the randomized trials. Lancet 2005;365:1687-1717.

2 Zeillinger R, Kury F, Czerwenka K, et al.: HER-2 amplification, steroid receptors and epidermal growth factor receptor in primary breast cancer. Oncogene 1989;4:109-114.

3 Slamon DJ, Leyland-Jones B, Shak S, et al.: Concurrent administration of anti-HER2 monoclonal antibody and first-line chemotherapy for HER2overexpressing metastatic breast cancer. A phase III, multinational, randomized controlled trial. $\mathrm{N}$ Engl J Med 2001;344:783-792.
4 Romond EH, Perez EA, Bryant J, et al.: Trastuzumab plus adjuvant chemotherapy for operable HER2-positive breast cancer. N Engl J Med 2005; 353:1673-1684.

5 Piccart-Gebhart MJ, Procter M, Leyland-Jones B, et al.: Trastzumab after adjuvant chemotherapy in Her2-positive breast cancer. N Engl J Med 2005; 353:1659-1672.

6 Münchner Krebsexperte beklagt Unterversorgung von Brustkrebs-Patientinnen. Der Spiegel 43/2005: October 22, 2005; http://www.spiegel.de/spiegel/ vorab/0,1518,381043,00.html
7 'Der zielgerichtete Einsatz ist das Problem', Profil 34/August 2005.

8 'Das ist enorm', Der Spiegel 43/2005: October 24 2005; http://www.spiegel.de/spiegel/0,1518,381161,00 html

9 Joensuu H, Kellokumpu-Lehtinen PL, Bono P, Alanko T, Kataja V, Asola R, Utriainen T, Kokko $\mathrm{R}$, Blanco G, Isola J: Trastuzumab in combination with docetaxel or vinorlebine as adjuvant treatment of breast cancer: the FinHer trial. SABCC, Abstr 2,2005 . 